【电子与信息科学／Electronics and Information Science】

\title{
网络多媒体数据中與情关联主题的挖掘方法
}

\author{
刘润奇 ${ }^{1}$ ，贺兴时 ${ }^{1}$ ，南夷非 ${ }^{2}$ ，王 博 $^{2}$
}

1) 西安工程大学理学院 陕西西安 $710048 ； 2$ ) 西安交通大学智能网络与网络安全教育部重点实验室 陕西西安 710049

摘 要: 如何高效地从图像、视频等多媒体数据中挖掘网络舆情事件的关联主题给网络興情的有效监 管带来了重大挑战. 研究图像和视频截图等多媒体数据中文本信息的抽取方法，并在此基础上实现舆情关 联主题的检测. 选择新浪微博中的 3 个典型與情事件为研究对象, 设计网络爬虫收集事件中的文本、图像 和视频多模态数据; 采用连接文本提议网络 (connectionist text proposal network, CTPN) 的文字检测算法实现 文本信息定位，利用 DenseNet 网络和连接时序分类 (connectionist temporal classification, CTC) 相结合的方法 进行文本提取; 提出多粒度潜在狄利克雷分布 (multi granularity-latent Dirichlet allocation, MG-LDA) 和 jieba 分词相结合的舆情关联主题提取方法. 实验结果表明，所提出的方法可准确提取多媒体数据中不同格式、 不同分辨率、不同颜色、不定位置和不同角度的文本信息，为精确把握與情演化态势提供有力的数据支撑.

关键词: 模式识别; 图像处理; 微型博客; 新浪微博; 多媒体数据; 文本检测; 文本提取; 主题识别; 舆情监管

中图分类号：TP393.3 文献标志码：A doi：10.3724/SP. J. 1249.2020.01072

\section{Mining method of public opinion related topic in network multimedia data}

\section{LIU Runqi ${ }^{1}$, HE Xingshi ${ }^{1}$, NAN Yifei ${ }^{2}$, and WANG Bo ${ }^{2}$}

1) School of Science, Xi'an Polytechnic University, Xi'an 710048, Shaanxi Province, P. R. China

2) Ministry of Education Key Lab for Intelligent Networks and Network Security, Xi'an Jiaotong University, Xi'an 710049, Shaanxi Province, P. R. China

\begin{abstract}
Social media has become the platform for rumors rapid propagation, more and more users adopt the pictures and videos to express their opinions in order to avoid being detected by text-based approaches, which has greatly affected the efficiency of online public opinion monitoring. For tackling the above-mentioned problem, this paper mainly studies how to extract the related opinions from the multimedia network data. Firstly, three typical events in Sina weibo are selected as the research targets, in which the web crawler is designed to collect the multimedia data. Secondly, the text detection algorithm based on connectionist text proposal network (CTPN) is employed to perform the text localization, and then a fusion method by combining DenseNet and connectionist temporal classification ( CTC) is employed to perform text extraction. Finally, an effective algorithm by combining multi granularity-latent Dirichlet allocation (MG-LDA) and jieba is proposed to accurately identify the related topics from the extracted text. The experimental results show that the proposed method can accurately extract the texts from
\end{abstract}

Received: 2019-04-06; Accepted: 2019-06-17

Foundation: Natural Science Basic Research Program of Shaanxi Province (2014JM1006) ; Natural Soft Science Research Program of Shaanxi Province (2014KRM2801); Key R \& D Program Projects of Shaanxi Province (2018KW-021)

Corresponding author: Professor HE Xingshi. Email: xsh1002@126.com

Citation: LIU Runqi, HE Xingshi, NAN Yifei, et al. Mining method of public opinion related topic in network multimedia data [ $\mathrm{J}$ ]. Journal of Shenzhen University Science and Engineering, 2020, 37(1): 72-78. (in Chinese) 
multimedia with different formats, resolutions and colors, and can also extract the texts with different rotating angles. Our research provides the solid foundations for online public opinion monitoring.

Key words : pattern recognition; image processing; microblog; Sina weibo ; multimedia data ; text detection; text extraction; subject recognition; public opinion supervision

近年来, 微博和论坛等新媒体的快速发展极大 方便了人们的生活, 但也为网络谣言和與情的快速 传播提供了温床, 给社会稳定和安全带来了巨大挑 战 ${ }^{[1]}$. 据不完全统计, 在中国目前微博月活跃用户 共 3.76 亿人，日活跃用户达到 1.65 亿人，每天产 生的帖子不计其数. 随着 $5 \mathrm{G}$ 网络的推行, 会进一 步提升网络使用的便捷度，加快與情事件的传播速 度. 与此同时，越来越多的與情事件依托图片和视 频等多媒体文件进行传播以躲避传统的基于文本的 舆情事件检测，本课题组在 2018 年 7-9 月对新浪 微博中的博文进行了分析，发现 $55 \%$ 的博文包含图 片信息, $35 \%$ 的博文包含视频信息,这些异构的多模 态数据给與情的有效监管造成了较大的困难.

舆情事件在近两年被大量研究. 梁冠华等 ${ }^{[2]}$ 将 舆情划分为潜伏期、发生期、持续期和恢复期，并 结合不同阶段的特征，设计了涵盖與情防范风险、 发生风险、传播风险和消退风险的指标体系，综合 评估與情的演化态势. 陈建敏等 ${ }^{[3]}$ 也从指标体系出 发, 提出涉军网络與情安全评估指标体系的内容构 成，具体包括发布者指标、受众指标、激体指标、 本体指标和传播指标，综合评估涉军與情的评估方 法. 丁㤹春等 ${ }^{[4]}$ 根据 “帖子-主题” 二模网络模型 和关键词以及社区属性对网络與情态势进行评估. 郑鬼等 ${ }^{[5]}$ 提出一种网络與情热点信息的自动发现方 法. 该方法对文本进行分词和词频统计，从词频表 中去除停用词得到突发事件热点信息关键词列表, 对网络信息及时检索，为突发事件应急决策提供技 术支持. 张艳丰等 ${ }^{[6]}$ 采用扎根理论提取指标要素, 通过直觉模糊推理算法和模糊综合评判法进行网络 舆情监测预警. 杨频等 ${ }^{[7]}$ 提出一种网络與情的定量 分析方法，该方法将对于某话题的文本集合作为输 人, 输出一个实数表示文本中所表达的观点倾向程 度. 陈星宇等 ${ }^{[8]}$ 从客户的参与、认知和情感 3 个维 度出发, 根据 3 维手动预定义关键词, 并基于这些 关键词找到特征客户, 最终以特征客户作为训练 集, 获得更多关于 3 个维度内容客户特征的关键 词，以达到进一步细分客户的目的. 这些方法的提 出一定程度解决了网络與情的监控问题. 如何在文 本信息检测的基础上，进一步处理图片和视频等多
媒体数据的检测, 是一个亟需解决的问题. 为此, 本研究提出结合深度神经网络的多媒体文本信息识 别方法, 并在此基础上提出了多粒度潜在狄利克雷 分布 (multi-granularity latent Dirichlet allocation, MGLDA $)^{[9]}$ 与 jieba 分词 ${ }^{[10]}$ 相结合的與情关联主题抽取 方法, 以期挖掘社交平台多媒体多数据中蕴含的與 情事件.

\section{1 识别方法框架}

本研究选择新浪微博 (http://weibo. com/) 上近 期关于性侵、疫苗和网贷 3 个社会反响强烈的與情 事件为研究对象, 采用 Scrapy 框架设计爬虫实现这 些事件中文本、图片和视频等多媒体数据的爬取. 通过分析收集到的数据，发现文本数据占 $64 \%$ ，图 片和视频数据占 $36 \%$ ，且绝大部分图片和视频包含 文本信息. 因此, 为便于处理, 将视频分帧, 并与图 片数据一并处理. 由于许多图片的分辨率严重失衡, 如低至 $365 \times 16367$ 像素, 可将其分割为 $1000 \times$ 1000 像素的固定图像块. 同时, 删除一些无文字 的图和严重失真的无效图, 以保证数据集的可靠性.

分析数据特点后发现，社交媒体中的图片具有 独特的特征, 如文本信息多有不同角度的旋转, 且 文字样式和颜色多变. 因此, 为提升不同颜色、不 同角度的文本识别精度，需先对偏转的文字进行水 平处理, 对彩色文字进行灰度处理. 其中, 特殊的 白色字体需进行反转处理. 最后, 结合所提取的文 本信息, 采用 MG-LDA 和 jieba 分词相结合的方法, 挖掘图片中蕴含的舆情主题. MG-LDA 比传统的 LDA (latent Dirichlet allocration) ${ }^{[11]}$ 主题识别方法更 易发现全局主题中的局部特征，即能够提取更多文 本中的有效信息. jieba 分词则是一款十分优秀的开 源分词引擎，它结合了基于字符串匹配的算法和基 于统计的算法，采用动态规划查找最大概率路径, 找出基于词频的最大概率组合，可以在分词速度快 的同时, 保持较高的分词精度. 结果表明, 本研究 方法可有效实现不同格式多媒体数据中不同颜色、 不同角度、不同位置的文本识别与提取，并在此基 础上实现與情关联主题的挖掘, 有效提高多媒体数 
据中舆情主题的监管效率.

多媒体数据中的网络與情事件关联主题检测模 型框架如图 1, 对应步骤的具体描述为:

1) 数据收集与预处理. 结合自媒体的特征, 在 Scrapy 框架下设计网络爬虫，收集包括文本、图 片和视频等多模态数据, 对视频数据进行离散化处 理, 形成图片.

2) 文本信息识别. 使用连接文本提议网络 ( connectionist text proposal network, CTPN $)^{[12-13]}$ 方法 实现图片数据中文字的检测, 用密集卷积神经网络
(DenseNet) 和连接时序分类 (connectionist temporal classification，CTC) 结合的方法进行文字识别, 通过图片切割、水平调整、灰度处理等方法实现不 同分辨率、不同角度和不同颜色的文本提取.

3) 关联主题识别. 结合 2) 中提取的文本特 征, 采用 jieba 模块进行分词处理, 并使用 MG-LDA 对其进行主题识别.

4）與情演化趋势管理. 深度融合图片数据中 关联主题的识别结果和文本数据中的识别结果, 以 期更准确掌握與情的演化态势.

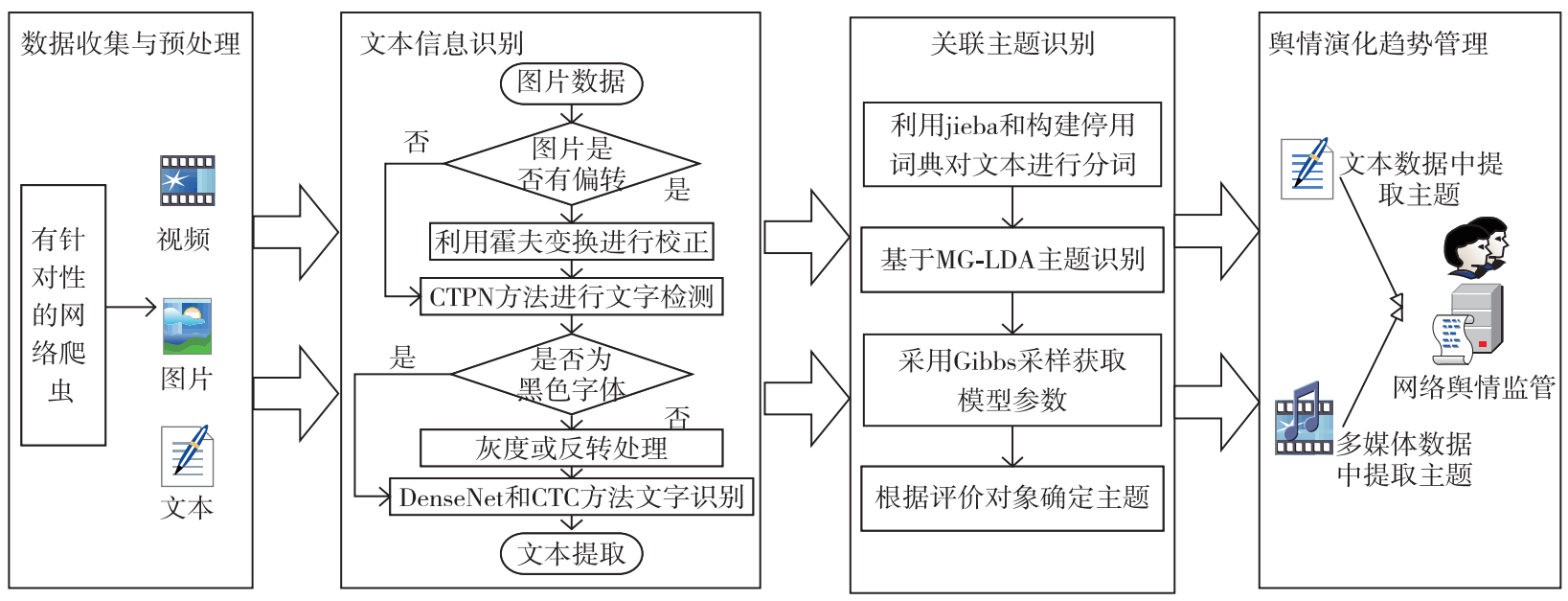

图 1 舆情检测框架

Fig. 1 (Color online) Public opinion detection framework

\section{2 多媒体数据中文字识别方法}

\section{1 具有旋转角度的文字定位方法}

传统的文字定位方法大多采用 bottom-up，即先 用滑动窗口等方法提取候选区域，再用支持向量机 ( support vector machine, SVM) 和卷积神经网络 (convolutional neural network, CNN) 等算法做字符 分类器，最后做字符聚类、字符分组和字符切割等 使其形成文本线. 但这种方法不够鲁棒、候选区域 过多、需人工干涉，且易造成误差积累. $\mathrm{HE}$ 等 $^{[14]}$ 提出 top-down 方法，通过先检测文本区域再测出文 本线来提高识别准确率. 随着深度神经网络的发 展，TIAN 等 ${ }^{[15]}$ 提出新型文字定位方法 CTPN，结 合了 CNN 和 RNN 优点. 深度学习为图像中文本信 息的抽取奠定了良好的理论基础，提升了文本识别 的效率和准确度. 但是, 这种方法只对水平文字具 有较好的效果，对偏转的文字检测效果则欠佳. 本
研究通过在 CTPN 方法定位文字前先对图片进行旋 转处理, 再对文本区域进行水平化, 利用霍夫变换 检测直线对有偏转角度的图片进行校正，提升了文 本识别方法的鲁棒性. 具有旋转角度的文字定位方 法的操作流程如图 2.

\section{2 多颜色文字提取方法}

DenseNet 是一种具有密集连接的卷积神经网 络. 在该网络中，任意两层之间都有直接的连接， 每层的输人都是前面所有层输出的并集，而该层所 学习的特征图也会被直接传给其后面所有层作为输 人. DenseNet 与 ResNet 的主要区别在于后者是求 和, 而前者是做拼接, 这种网络与传统网络相比更 能提升信息和梯度在网络中的传输效率.

$\mathrm{CTC}^{[12-13]}$ 主要用来解决时序类数据的分类问 题, 即实现输人序列到输出序列的映射. 本研究采 用密集卷积神经网络 DenseNet 提取文字图像底层特 征, 加人时域连接模型 CTC 解码获得识别的文字. 但是该模型是在黑色标准字体下训练得到的，对于 


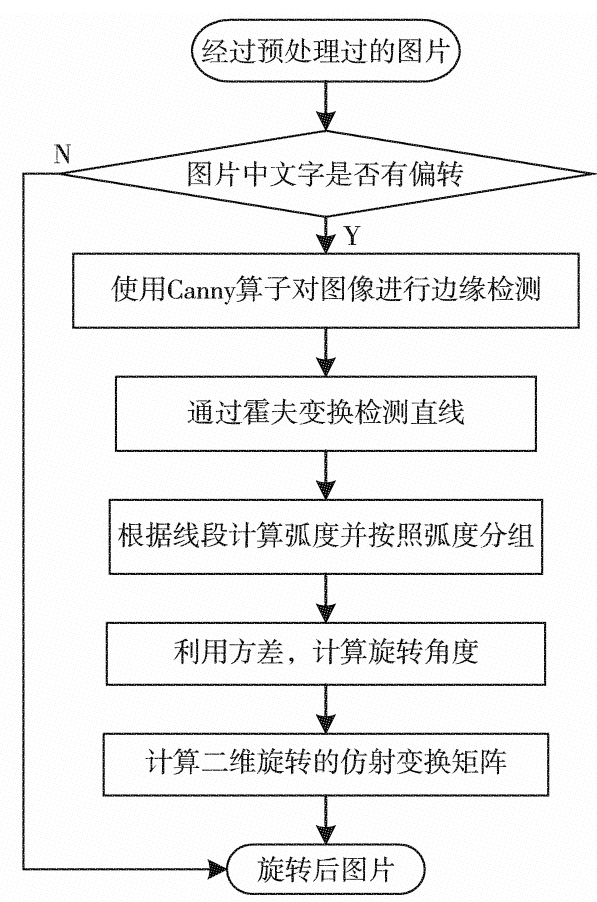

图 2 校正偏转角度文字流程

Fig. 2 Flowchart of correction of text deflection angle

包含其他颜色文字的图片处理效果欠佳. 据此我们 首先对图片进行灰度处理, 使其三维像素矩阵转换 为二维. 对于白色字体进一步对其进行反转处理, 即像素值由 0 转为 255 , 依此来提升不同颜色字图 的识别效果. 多颜色文字提取的操作流程见图 3 .

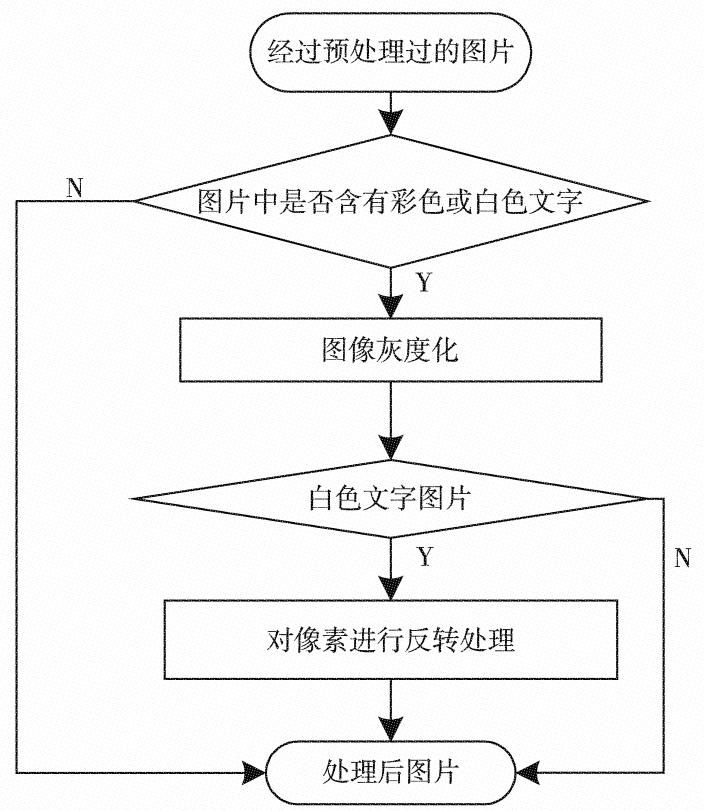

图 3 处理有色文字流程

Fig. 3 Flowchart of colored text processing
3 基于 MG-LDA 与 jieba 分词的图像 主题识别方法

由于本研究采集到的图片是针对同一类型事件 的, 识别后的文本数据在文本结构特征上更易出现 难以挖掘主题的现象，所以采用 MG-LDA ${ }^{[9]}$ 模型建 模，通过区分全局主题和局部主题，可更好地发现 图片中讨论的與情要素. 根据文献 [9]方法获得模 型参数, 并基于 jieba 和构建停用词典对词语进行 分词处理. 基于 MG-LDA 的图像主题识别方法的具 体流程见图 4.

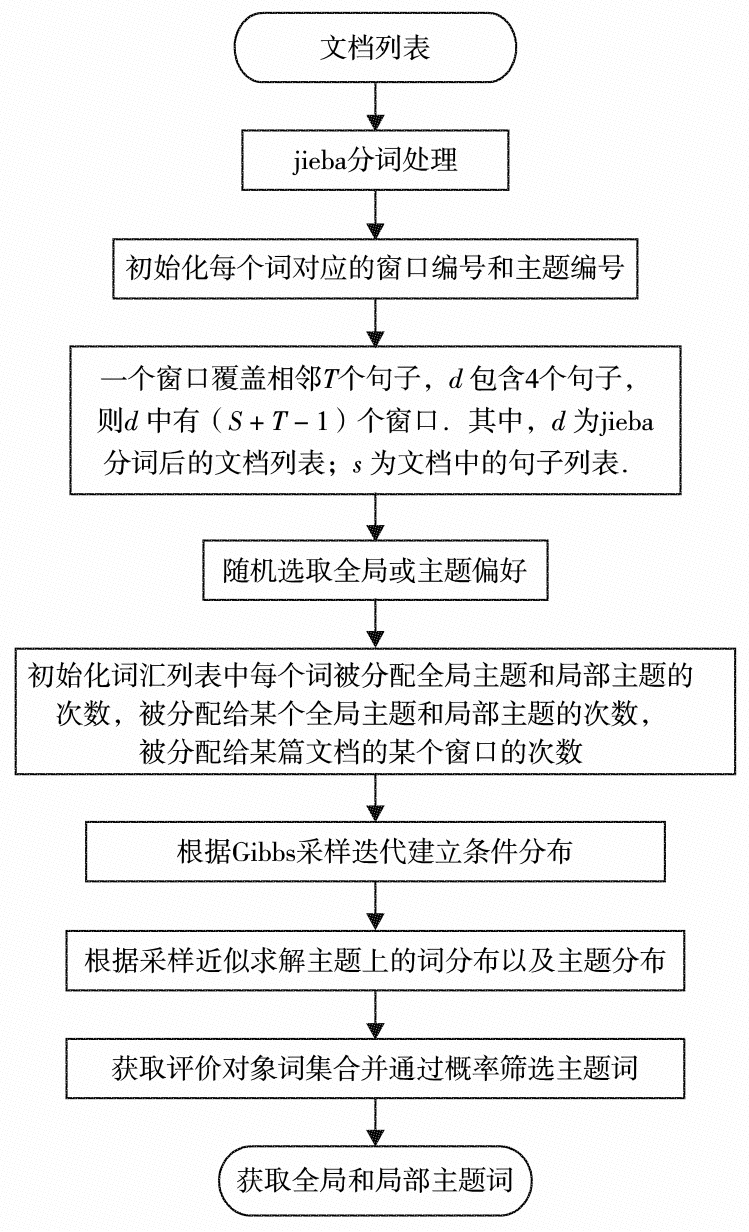

图 4 MG-LDA 主题识别流程

Fig. 4 Flowchart of MG-LDA topic recognition

4 实验与分析

\section{1 数据收集}

本研究选择新浪微博中近期 3 个社会舆论反响 强烈的敏感话题为研究对象，爬取收集性侵、疫苗 
和网贷 3 个热点事件下的文本、图片和视频数据, 结果如表 1. 其中，文本表示博文的数量; 图片表 示博文中包含图片的数量; 彩色文本表示包含有彩 色文字图片的数量; 歪斜文本表示包含有偏转角度 文本图片的数量. 由表 1 可见，歪斜文本和彩色文 本占比不小, 需要根据它们的特征进行适当的处 理，才能取得理想的识别效果.

\section{表 1 舆情事件数据集统计}

Table 1 Data set of public opinion events

\begin{tabular}{lcccc}
\hline 事件 & 文本/篇 & 图片/张 & 彩色文本/张 & 歪斜文本/张 \\
\hline 性侵 & 2158 & 1791 & 652 & 135 \\
疫苗 & 2303 & 1163 & 503 & 92 \\
网贷 & 2616 & 1465 & 579 & 97 \\
\hline
\end{tabular}

\section{2 文本提取结果分析}

采用本研究方法, 从图片中获取和直接获取的 文本, 处理后的数据集如表 2. 其中, 文本数据为 直接从文本数据中提取的字符数量；图片数据为经 过对图片进行文本信息识别提取的字符总量. 由表 2 可见，图片中文本数据的占比超过 $50 \%$ ，表明对 图片中的文本数据进行识别和分析非常重要.

表 2 文本处理结果

Table 2 Text processing results 个

\begin{tabular}{cccc}
\hline 事件 & 文本数据 & 图片数据 & 合计 \\
\hline 性侵 & 243315 & 313042 & 556357 \\
疫苗 & 281388 & 242742 & 524130 \\
网贷 & 331370 & 337356 & 668726 \\
\hline
\end{tabular}

使用文本数据的提取率、准确率和算法的时间 复杂度 3 个指标评估算法性能. 文本数据的提取率 为能够从图片数据中准确识别并提取文本的比例, 用来衡量是否能够提取出图像数据中的文本数据. 文本信息提出的准确性为识别出文本信息的正确 率. 时间复杂度为处理一定数量的图片所需时间, 用来衡量所提方法的实用性. 对所收集到的 4419 张涉及 3 个與情的图片数据进行文本识别，结果发 现, 采用本研究方法对歪斜字体进行旋转处理和对 图片中存在的彩色字体进行灰度或反转处理, 提取 率为 $96.23 \%$, 准确率为 $95.31 \%$; 而不对图片做 任何处理的未改进方法, 提取率为 $50.23 \%$, 准确 率为 $65.23 \%$, 可见本研究方法可大幅提升识别方 法的提取率和准确率.
分别在中央处理器 (central processing unit, CPU) 和图形处理器 (graphics processing unit, GPU) 环境下进行时间复杂度分析，基于与 Python3. 6 相 匹配的 Anaconda 库，使用 TensorFlow、Keras、 OpenCV 等模块实现. 实验结果如图 5. 由图 5 可 见，在 GPU 环境下可以大幅度的压缩计算时间, 进一步满足了实效性的要求.

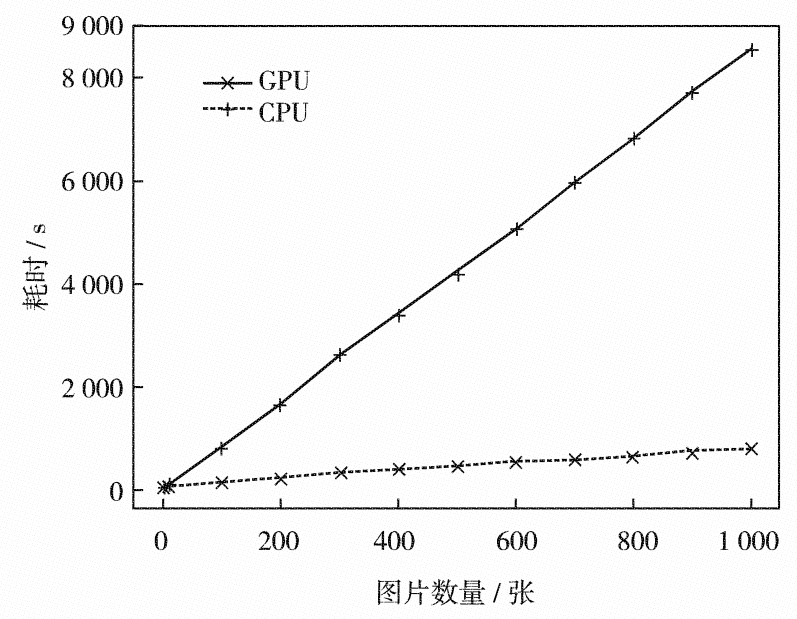

图 5 不同环境下时间复杂度对比

Fig. 5 Time complexity comparison in different environments

\section{3 关联主题结果分析}

采用 MG-LDA 模型分别对由图像数据中抽取的 文本和文本数据进行主题识别. 使用 Python 语言实 现 MG-LDA 模型, 参照文献 [16-17] 给出的模型参 数值, 并通过实验调整, 得到参数赋值为: 全局主 题分布的狄利克雷 (Dirichlet) 参数 $\alpha^{\mathrm{gl}}=0.6$, 局部 主题分布的狄利克雷参数 $\alpha^{\text {loc }}=0.6$, 全局单词分布 的狄利克雷参数 $\beta^{\text {gl }}=0.01$, 局部单词分布的狄利克 雷参数 $\beta^{\text {loc }}=0.001, \alpha_{g 1}^{\text {mix }}=0.01$, 局部主题先验分 布的非对称贝塔 (Beta) 参数 $\alpha_{\mathrm{loc}}^{\mathrm{mix}}=0.50$; 先验分布 的对称狄利克雷参数 $\gamma=0.01$. 全局主题数 $\mathrm{gl}=18$, 局部主题数 loc $=6$; 窗口中相邻句子数 $T=3$; 迭代 次数 $I=1000$. 识别后篮选出的全局主题和局部主 题中可做为舆情要素的词语如表 3 和表 4 . 由表 3 和表 4 可见, 由图像中识别的舆情要素可作为引发 网络與情的必要条件，但根据文本识别的主题词和 图像识别的主题词有所不同. 例如，在本研究针对 性侵事件的分析中，发现图片等多媒体数据中提取 的信息多和沉默、权利以及高官相关，比纯文本数 据蕴含了更多的舆情要素，社会关注的焦点往往会 在受害者或者事件中的强势群体，这就更加容易形 
成舆情 ${ }^{[18]}$. 可见，综合分析图像和文本等多模态 数据, 可更加完善的获取舆情关联要素, 这对于监 控舆情演化趋势十分重要.

\section{表 3 文本数据主题识别结果}

Table 3 Text data topic recognition results

\begin{tabular}{|c|c|c|}
\hline $\begin{array}{l}\text { 與情 } \\
\text { 事件 }\end{array}$ & 层级 & 與情要素 \\
\hline \multirow{2}{*}{ 性侵 } & 全局 & $\begin{array}{l}\text { 孩子、新闻、社会、指控、幼儿园、身体、爆 } \\
\text { 料、性骚扰、围观 }\end{array}$ \\
\hline & 局部 & 人物、亲生、收养、神职人员、志愿者 \\
\hline \multirow[t]{2}{*}{ 疫苗 } & 全局 & $\begin{array}{l}\text { 医师、生命、制度、真相、生育、健康、狂犬 } \\
\text { 病、股票、合格 }\end{array}$ \\
\hline & 局部 & 食品药品、监督管理、免职、围观、感染 \\
\hline \multirow[t]{2}{*}{ 网贷 } & 全局 & $\begin{array}{l}\text { 网络、大学生、诈骗、造假、黑户、乱象、高 } \\
\text { 利贷、裸贷、平台 }\end{array}$ \\
\hline & 局部 & P2P、整治、风险、套路、业务 \\
\hline
\end{tabular}

\section{表 4 图像数据主题识别结果}

Table 4 Image data topic recognition results

\begin{tabular}{|c|c|c|}
\hline $\begin{array}{l}\text { 與情 } \\
\text { 事件 }\end{array}$ & 层级 & 與情要素 \\
\hline \multirow{2}{*}{ 性侵 } & 全局 & $\begin{array}{l}\text { 侵害、晚上、威胁、法庭、痛苦、沉默、女 } \\
\text { 性、教师、运动 }\end{array}$ \\
\hline & 局部 & 视频、媒体、猥鈴、权利、言论 \\
\hline \multirow[t]{2}{*}{ 疫苗 } & 全局 & $\begin{array}{l}\text { 司法、小孩、曝光、症状、检测、判决、政 } \\
\text { 策、狂犬病、王老吉 }\end{array}$ \\
\hline & 局部 & 长生、病毒、合格、过期、批号、接种 \\
\hline \multirow[t]{2}{*}{ 网贷 } & 全局 & $\begin{array}{l}\text { 贷款、保险公司、集资、P2P、理财、少女、 } \\
\text { 监管、非法、诈骗 }\end{array}$ \\
\hline & 局部 & 交易、期限、平台、涉嫌、刑事 \\
\hline
\end{tabular}

\section{结 语}

通过研究社交媒体图片的文本识别方法及其在 网络與情监管的应用, 结合所收集的图片的特征, 针对图片中因字体歪斜、字体颜色多变等特点给文 本提取带来的困难，提出了不规则图片数据中文本 的识别与提取方法, 并对所提文本的主题特征进行
了分析. 对收集到的新浪微博上关于性侵、疫苗和 网贷 3 个热点事件的关联文本和图像数据的提取和 主题识别结果表明，所提方法在社交媒体图片的文 本识别中具有较好的性能, 能够解决社交媒体图片 不规则字体的提取，为网络與情监管提供了有力的 技术支撑. 下一步，我们将主要分析如何识别一些 图片中存在的多语言文字，进一步挖掘图像中蕴含 的和网络舆情相关的语义信息.

基金项目：陕西省自然科学基础研究计划资助项目（2014JM1006); 陕西省自然软科学研究计划资助项目 (2014KRM2801); 陕西省重点研发计划资助项目 (2018KW-021)

作者简介: 刘润奇 (1994-), 西安工程大学硕士研究生. 研究方 向：网络與情事件的关联主题挖掘方法研究. E-mail: rliu164103@163.com

引文：刘润奇, 贺兴时, 南夷非, 等. 网络多媒体数据中舆情 关联主题的挖掘方法 $[\mathrm{J}]$. 深圳大学学报理工版, 2020 $37(1)$ : $72-78$

\section{参考文献／References:}

[ 1 ] 陈子鸥. 新媒体时代的舆情管理 [N]. 德宏团结报, 2019-05-28(002).

CHEN Ziou. Public opinion management in the new media age $[\mathrm{N}]$. Dehong Tuanjie Bao, 2019-05-28 (002). (in Chinese)

[ 2 ] 梁冠华, 鞠玉梅. 基于與情演化生命周期的突发事件 网络與情风险评估分析 $[\mathrm{J}]$. 情报科学, 2018，36 (10) : 48-53.

LIANG Guanhua, JU Yumei. The analysis of public opinion risk assessment of sudden events based on public relation life cycle $[\mathrm{J}]$. Information Science, 2018, 36 (10) : 38-53. (in Chinese)

[3] 陈建敏, 余遵成. 涉军网络與情安全评估指标体系研 究 $[\mathrm{J}]$ ．图书情报研究，2018，11(1)：29-36.

CHEN Jianmin, YU Zuncheng. A study of the indicator system for evaluating the safety of military network public opinions $[\mathrm{J}]$. Library and Information Research, 2018 , 11(1) : 29-36. (in Chinese)

[4] 丁戟春，王 楠，吴鈽婵媛. 基于关键词共现和社区 发现的微博热点主题识别研究 $[\mathrm{J}]$. 现代情报, 2018, $38(3)$ : $10-18$.

DING Shengchun, WANG Nan, WU Jingchanyuan. Hot topic detection of weibo based on keyword co-occurrence and community discovery $[\mathrm{J}]$. Journal of Modern Information, 2018, 38(3) : 10-18. (in Chinese)

[5]郑 魁, 疏学明, 袁宏永. 网络舆情热点信息自动发 现方法 $[\mathrm{J}]$. 计算机工程, 2010, 36(3) : 4-6.

ZHENG Kui, SHU Xueming, YUAN Hongyong. Hot spot information auto-detection method of network public 
opinion $[\mathrm{J}]$. Computer Engineering, 2010, 36(3) : 4-6. (in Chinese)

[6]张艳丰, 李 贺, 彭丽徽. 基于直觉模糊推理的网络 與情监测预警评估方法研究 $[\mathrm{J}]$ ．情报杂志，2017， 36(6) : 111-117, 172.

ZHANG Yanfeng, LI He, PENG Lihui. Research on network public opinion monitoring and early warning evaluation method based on intuitionistic fuzzy reasoning [J]. Journal of Intelligence, 2017, 36 (6) : 111-117, 172. (in Chinese)

[ 7 ] 杨 频, 李 涛, 赵 奎. 一种网络舆情的定量分析 方法 $[J]$. 计算机应用研究，2009，26(3)：272-274, 284.

YANG Pin, LI Tao, ZHAO Kui. Quantitative method for analyzing public opinions on Internet $[\mathrm{J}]$. Application Research of Computers, 2009, 26(3) : 272-274, 284. (in Chinese)

[8] 陈星宇, 周 展, 莫文俊, 等. 基于关键词挖掘的客 户细分方法 $[\mathrm{J}]$. 深圳大学学报理工版, 2017, 34 (3) : 300-305.

CHEN Xingyu, ZHOU Zhan, HUANG Wenjun, et al. A keyword-based mining method for customer segmentation [J]. Journal of Shenzhen University Science and Engineering, 2017, 34(3) : 300-305. (in Chinese)

[9] BURKHARDT S, KRAMER S. Online multi-label dependency topic models for text classification $[\mathrm{J}]$. Machine Learning, 2018, 107(5) : 859-886.

[10] 邢 彪, 根线切机多吉. 基于 jieba 分词搜索与 SSM 框架的电子商城购物系统 $[\mathrm{J}]$. 信息与电脑, 2018 (7) : 104-105.

XING Biao, Genrongqiejiduoji. E-commerce shopping system based on jieba participle search and SSM framework [J] . China Computer \& Communication, 2018(7) : 104105. (in Chinese)

[11] JELODAR H, WANG Yongli, YUAN Chi, et al. Latent Dirichlet allocation (LDA) and topic modeling: models, applications, a survey $[\mathrm{J}]$. Multimedia Tools and Applications, 2018: 1-43.

[12] GRAVES A, FERNÁNDEZ S, GOMEZ F, et al. Connectionist temporal classification: labelling unsegmented sequence data with recurrent neural networks
[C] // Proceedings of the 23rd International Conference on Machine Learning. Pittsburgh, USA: ACM, 2006: 369-376

[13] 张艺玮，赵一嘉，王馨悦，等. 结合密集神经网络与 长短时记忆模型的中文识别 $[\mathrm{J}]$ 。计算机系统应用， 2018, 27(11) : 35-41.

ZHANG Yiwei, ZHAO Yijia, WANG Xinyue, et al. Chinese recognition based on dense convolutional network and bidirectional long short-term memory model $[\mathrm{J}]$. Computer Systems \& Applications. 2018, 27 ( 11 ) : 3541. (in Chinese)

[14] HE Tong, HUANG Weilin, QIAO Yu, et al. Accurate text localization in natural image with cascaded convolutional text network [DB/OL]. (2016-05-31) [2019-0401]. https://arxiv. org/1603.09423. pdf

[15] TIAN Zhi, HUANG Weilin, HE Tong, et al. Detecting text in natural image with connectionist text proposal network $[\mathrm{C}] / /$ European Conference on Computer Vision. Amsterdam: Springer, 2016: 56-72.

[16] 缪裕青, 汪俊宏, 刘同来, 等. 图文融合的微博情感 分析方法 $[\mathrm{J}]$. 计算机工程与设计, $2019,40(4)$ ： 1099-1105.

MIAO Yuqing, WANG Junhong, LIU Tonglai, et al. Joint visual-textual approach for microblog sentiment analysis [J]. Computer Engineering and Design, 2019, 40 (4) : 1099-1105. (in Chinese)

[17] 彭 云，万常选，江腾蛟，等. 基于语义约束 LDA 的商品特征和情感词提取 $[\mathrm{J}]$. 软件学报, 2017，28 (3) : 676-693.

PENG Yun, WAN Changxuan, JIANG Tengjiao, et al. Extracting product aspects and user opinions based on semantic constrained LDA model [ J ]. Journal of Software, 2017, 28(3) : 676-693. (in Chinese)

[18] 钟杏梅. 网络與情传播规律及成因探析 $[\mathrm{J}]$. 公关世 界，2017(19)：34-39.

ZHONG Xingmei. Analysis of network public opinion on propagation model and cause [J]. Public Relations, 2017 (19) : 34-39. (in Chinese)

【中文责编：英 子; 英文责编: 木 柯】 